\title{
New results in conventional and exotic spectroscopy
}

2 Adriano Di Florio ${ }^{a, *}$

${ }_{3}^{a}$ Universitá degli Studi di Bari Aldo Moro and I.N.F.N. - Sezione di Bari

4 E-mail: adriano.di.florio@cern.ch

The present report summarises three recent CMS results in conventional and exotic spectroscopy. Firstly the search for a narrow resonance decaying to $\Upsilon(1 S) \mu^{+} \mu^{-}$performed with $p p$ collision data collected in 2016 with the CMS detector at the LHC in proton-proton collisions at $\sqrt{s}=13 \mathrm{TeV}$, corresponding to an integrated luminosity of $35.9 \mathrm{fb}^{-1}$. A tetraquark $(b b \bar{b} \bar{b})$ search is performed for masses in the vicinity of four times the bottom quark mass, between 17.5 and $19.5 \mathrm{GeV}$, while a generic search for other resonances is performed for masses between 16.5 and $27 \mathrm{GeV}$. No significant excess of events compatible with a narrow resonance is observed in the data. Limits on the production cross section times branching fraction of its decay to four muons via an intermediate $\Upsilon(1 S)$ resonance are set as a function of the resonance mass.

In the second part, the study of $B^{+} \rightarrow J / \psi \bar{\Lambda} p$, performed using $p p$ collision data collected in 2012 at $\sqrt{s}=8 \mathrm{TeV}$, corresponding to an integrated luminosity of $19.6 \mathrm{fb}^{-1}$, is presented.

5 The ratio of branching fractions $\mathcal{B}\left(B^{+} \rightarrow J / \psi \Lambda p\right) / \mathcal{B}\left(B^{+} \rightarrow J / \psi K(892)^{+}\right)$is measured to be $(1.054 \pm 0.057$ (stat) \pm 0.035 (syst) $\pm 0.011(\mathcal{B})) \%$. In addition the invariant mass distributions of the $J / \psi \Lambda, J / \psi p$, and $\Lambda p$ systems produced in the $B^{+} \rightarrow J / \psi \bar{\Lambda} p$ decay are investigated. Using a model-independent angular amplitude analysis approach, it is shown that the observed invariant masses distributions are consistent with the contributions from excited kaons decaying to the $\Lambda p$ system.

Finally the study of excited $\Lambda_{b}^{0}$ baryons is reported, based on a data sample collected in 2016-2018 at a center-of-mass energy of $13 \mathrm{TeV}$, corresponding to an integrated luminosity of up to $140 \mathrm{fb}^{-1}$. The existence of four excited $\Lambda_{b}^{0}$ states: $\Lambda_{b}(5912)^{0}, \Lambda_{b}(5920)^{0}, \Lambda_{b}(6146)^{0}$ and $\Lambda_{b}(6152)^{0}$ decaying to $\Lambda_{b}^{0} \pi^{+} \pi^{-}$is confirmed. Also a broad excess of events in the $\Lambda_{b}^{0} \pi^{+} \pi^{-}$mass distribution in the region of $6040 \div 6100 \mathrm{MeV}$ is observed.

BEAUTY2020

21-24 September 2020

Kashiwa, Japan (online)

${ }^{*}$ Speaker 


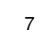

\section{Search for resonances decaying to $\Upsilon(1 S) \mu^{+} \mu^{-}$}

Quarkonium pair production is an important probe of both perturbative and nonperturbative processes in quantum chromodynamics. In [1] CMS measured the cross section for $\Upsilon(1 S)$ pair production with both mesons decaying to $\mu \mu$ and it also performed a search for a narrow resonance decaying to $\Upsilon(1 S) \mu^{+} \mu^{-}$. Such a resonance could indicate the existence of a tetraquark that is a bound state of two $b$ quarks and two $\bar{b}$ antiquarks. The search for $b b \bar{b} \bar{b}$ tetraquarks is performed for $17.5 \leq m(4 \mu) \leq 19.5 \mathrm{GeV}$, while the generic search for a narrow resonance is done for $16.5 \leq m(4 \mu) \leq 27 \mathrm{GeV}$. In both cases the $\Upsilon(1 S)$ pair production serves as a reference, since the final state is the same and a similar event selection is used. The measurement of the $\Upsilon(1 S)$ pair production cross section is reported in another conference contribution. The paper relies on protonproton collision data collected at $\sqrt{s}=13 \mathrm{TeV}$ by the CMS detector in $2016\left(\mathcal{L}_{\text {int }}=35.9 \mathrm{fb}^{-1}\right)$.

Events are selected with a trigger that requires the presence of three muons, among which two must have an invariant mass compatible with a $\Upsilon$ resonance $(8.5<m(\mu \mu)<11.4 \mathrm{GeV})$ and the dimuon vertex fit probability must be greater than $0.5 \%$. Each event is then required to have four reconstructed muons with $p_{T}(\mu)>2 \mathrm{GeV}$ and $|\eta(\mu)|<2.4$. For each event, the combination of four muons with the largest $\chi^{2}$ probability is chosen and a $p_{T}(\mu) 2.5 \mathrm{GeV}$ cut is applied. The event is discarded if one of the two alternative opposite sign muon pairs has an invariant mass either compatible with a $J \psi$ particle or lower than $4 \mathrm{GeV}$.

For the resonance search, $m(\Upsilon(1 S))$ is also required to be within $2 \sigma$ of the experimental resolution (between 0.06 and $0.15 \mathrm{GeV}$ ) from the $\Upsilon(1 S)$ nominal mass [2]. Assuming that the resonant state decays into two muons and a $\Upsilon(1 S)(\rightarrow \mu \mu)$, the signal mass resolution is improved by using the observable [3]: $\tilde{m}_{4 \mu}=m_{4 \mu} m_{\mu \mu}+m_{\Upsilon(1 S)}$; where $m_{4 \mu}$ is the invariant mass of the four leptons, $m_{\mu \mu}$ the invariant mass associated with the $\Upsilon(1 S)$ candidate, and $m_{\Upsilon(1 S)}$ the nominal mass of the $\Upsilon(1 S)$ particle $\left(9.46 \mathrm{GeV}\right.$ [2]). This $\tilde{m}_{4 \mu}$ has a resolution about $50 \%$ better than $m_{4 \mu}$ for signal events.

Multiple models are take into account for the narrow resonance: a bottomonium state with the properties of the $\chi_{b 1}(1 P)$, assuming a phase-space decay to a $\Upsilon(1 S)$ meson and a pair of muons (simulated with PYTHIA 8.226 generator [6]; a scalar, pseudoscalar and a spin-2 particle produced in gluon fusion (simulated with JHUGEN generator [7]). For each model, four resonance mass values are simulated: $14,18,22$, and $26 \mathrm{GeV}$. The signal distributions are parameterised by the sum of two Gaussian functions with the same mean and parameters that are determined from the simulated signal samples.

One of the background components comes from the $\Upsilon(1 S) \Upsilon(1 S)$ process. It is modelled as the product of a sigmoid function and an exponential function with a negative exponent. The nominal model for or the invariant mass of this background is taken as an average between the DPS (double parton scattering) and SPS (single parton scattering) templates, with the DPS fraction estimated, in the same paper, from the pair production cross section measurement $\left(f_{D P S}=0.39 \pm 0.14\right)$. The number of $\Upsilon(1 S) \Upsilon(1 S)$ events in the signal region is extracted from a $2 \mathrm{D}$ fit to the invariant masses of the two muon pair without applying the acceptance and efficiency corrections. Only events with $13<\tilde{m}_{4 \mu}<28 \mathrm{GeV}$ are retained and no rapidity criteria are applied for the reconstructed $\Upsilon(1 S)$ candidates. The yield is measured to be $78 \pm 13$ events. The requirement that the mass of a dimuon pair is compatible with the mass of a $\Upsilon(1 S)$ meson within $2 \sigma$ is not applied to extract 

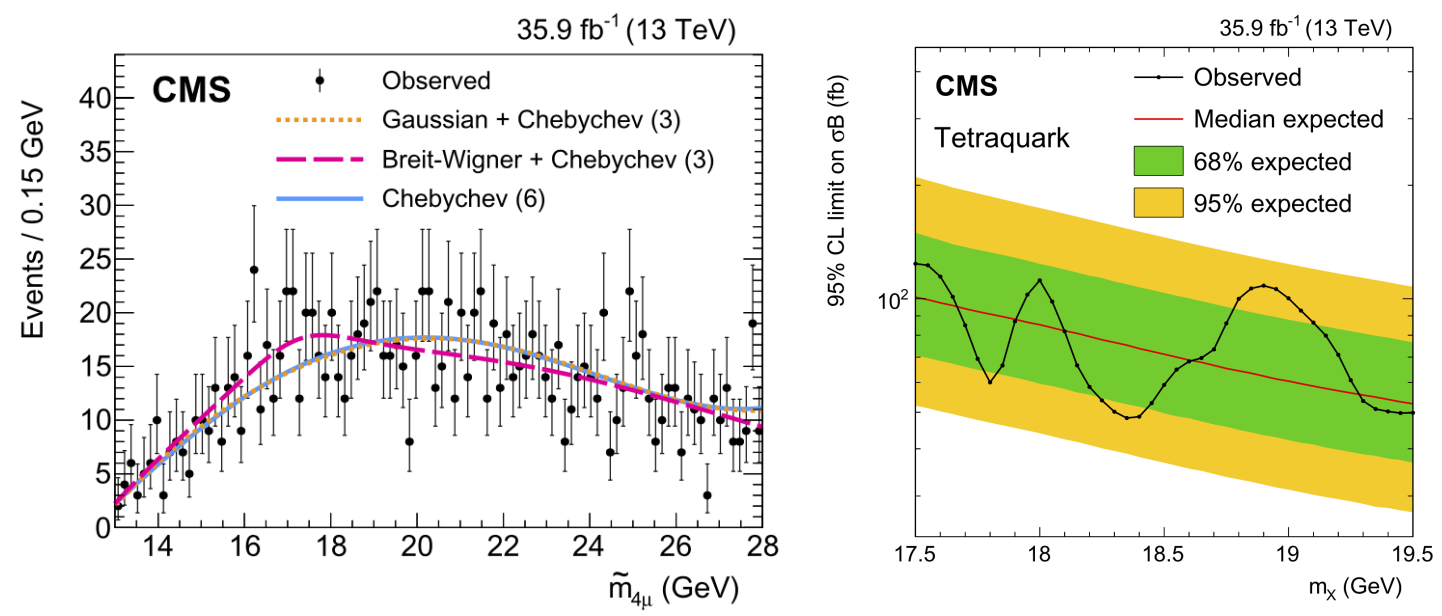

Figure 1: Left: The $\tilde{m}_{4 \mu}$ distribution from data and the results of the fit in the resonance search. An example signal, with $1 \sigma$ significance, is shown for the tetraquark model with a mass of $19 \mathrm{GeV}$. Right: Upper limits at $95 \% \mathrm{CL}$ on the product of the cross section and branching fraction for a tetraquark, where $\sigma$ denotes the production cross section of the resonance, and $\mathcal{B}=\mathcal{B}(X \rightarrow \Upsilon(1 S) \mu \mu) \cdot \mathcal{B}(\Upsilon(1 S) \rightarrow \mu \mu)$.

the yield because the $2 \mathrm{D}$ fit relies on the mass tails to estimate the combinatorial background. Since the efficiency of this criterion is $95 \%$ in both the SPS and DPS $\Upsilon(1 S) \Upsilon(1 S)$ simulations, the $\Upsilon(1 S) \Upsilon(1 S)$ yield in the signal region is corrected to $74 \pm 13$.

The combinatorial background in the $\tilde{m}_{4 \mu}$ spectrum is obtained by fitting the data in the signal region. Several generic functions are used to parameterise this smooth background: Chebychev polynomials of various orders, the sum of a Gaussian and a Chebychev polynomial, the sum of a Breit-Wigner and a Chebychev polynomial. The compatibility of such forms with the smooth $\tilde{m}_{4 \mu}$ spectrum of the combinatorial background is checked and confirmed using a control region where the vertex fit $\chi^{2}$ probability of the four muons is in the range of $10^{-10}-10^{-3}$. The parameters of the functions, as well as the choice of the functional form, are freely floating in the fit to the signal region. Multiple sources of systematic uncertainty are taken into account, such as, citing the most important: the integrate luminosity estimation, the trigger and the muon identification efficiencies, the signal and background modelling and the limited size of the simulated samples.

The $\tilde{m}_{4 \mu}$ distribution in the signal region of the resonance search is shown in the left panel of Fig. 1. The background and example signal components are shown using their best-fit shapes and normalisations. No significant narrow excess of events is observed above the background expectation.

Upper limits on the product of the production cross section of a resonance and the branching fraction to a final state of four muons via an intermediate $\Upsilon(1 S)$ resonance are set at $95 \%$ confidence level (CL) using the modified frequentist construction CLs in the asymptotic approximation [4][5], separately for each signal model. Masses between 17.5 and $19.5 \mathrm{GeV}$ are probed in the context of the tetraquark search (see Figure 1), using the bottomonium model, whereas the limits in the extended mass range $16.5 \div 27 \mathrm{GeV}$ are set for the generic search. The largest excess is observed for a resonance mass of $25.1 \mathrm{GeV}$, and has a local significance of $2.4 \sigma$ for the scalar signal hypothesis. In conclusion, no excess of events compatible with a signal is observed in the four-muon invariant mass spectrum and this statement would need to be reassessed with the full Run2 statistics. 


\section{Study of the $B^{+} \rightarrow J / \psi \bar{\Lambda} p$ decay}

The $B^{+} \rightarrow J / \psi \bar{\Lambda} p$ decay is the first observed example of a $B$ meson decay into baryons and a charmonium state. In [8] a study is reported of the $B^{+} \rightarrow J / \psi \bar{\Lambda} p\left(J / \psi \rightarrow \mu^{+} \mu^{-}, \Lambda \rightarrow p \pi^{+}\right)$decay using a data sample of $p p$ collisions collected by the CMS experiment in 2012 at $\sqrt{(} s)=8 \mathrm{TeV}$ $\left(\mathcal{L}_{\text {int }}=19.6 \mathrm{fb}^{-1}\right)$. The decay $B^{+} \rightarrow J / \psi K^{*+}\left(K^{*+} \rightarrow K_{\mathrm{S}}^{0} \pi^{+} \rightarrow \pi^{+} \pi^{-} \pi^{+}\right)$is chosen as the normalisation channel (where $K^{*+}$ denotes the $K^{*}(892)^{+}$particle). The ratio of the branching fractions is measured as: $\frac{\mathcal{B}\left(B^{+} \rightarrow J / \psi \bar{\Lambda} p\right)}{\mathcal{B}\left(B^{+} \rightarrow J / \psi K^{*+}\right)}=\frac{N\left(B^{+} \rightarrow J / \psi \bar{\Lambda} p\right) \mathcal{B}\left(K^{*+} \rightarrow K_{\mathrm{S}}^{0} \pi^{+}\right) \mathcal{B}\left(K_{\mathrm{S}}^{0} \rightarrow \pi^{+} \pi\right) \epsilon\left(B^{+} \rightarrow J / \psi K^{*+}\right)}{N\left(B^{+} \rightarrow J / \psi K^{*+}\right) \mathcal{B}\left(\bar{\Lambda} \rightarrow \bar{p} \pi^{+}\right) \epsilon\left(B^{+} \rightarrow J / \psi \bar{\Lambda} p\right)}$; where $N$ and $\epsilon$ correspond to the total yield and the total efficiency of the decay, respectively. Data were collected with a dedicated trigger, optimised for the selection of $b$ hadrons decaying to $J / \psi\left(\rightarrow \mu^{+} \mu^{-}\right)$. The final $B^{+}$candidate is built combining the $J / \psi$ candidate with a positively charged particle track, assumed to be a $p$ track, and a $\bar{\Lambda}$ candidate, formed from displaced two prong vertices under the assumption of the $\bar{\Lambda} \rightarrow p \pi^{+}$decay [9]. A further selection on the displacement and the pointing angle is applied, in order to remove most of the combinatorial background. Contamination from $K_{S}^{0} \rightarrow \pi^{-} \pi^{+}$decays is removed.

The normalisation decay channel $B^{+} \rightarrow J / \psi K^{*+}$ candidates are built using the same reconstruction chain. The distribution of $M\left(B^{+} \rightarrow J / \psi \bar{\Lambda} \bar{p}\right)$ is shown in Figure 2 resulting in a signal yield of $452 \pm 23$ events. To extract the $K^{*+}$ meson contribution, $s P l o t$ technique [10] is used with $M\left(J / \psi K_{S}^{0} \pi^{+}\right)$as discriminating variable. The $B^{+} \rightarrow J / \psi K^{*+}$ signal yield $(20863 \pm 357)$ is then extracted integrating the signal fit over $\pm 50 \mathrm{MeV}$ around the $K^{*+}$ mass.

The efficiency for detecting and identifying the $B^{+}$decays is calculated using simulated signal samples. The efficiency ratio is found to be $\epsilon\left(B^{+} \rightarrow J / \psi K^{*+}\right) / \epsilon\left(B^{+} \rightarrow J / \psi \bar{\Lambda} p\right)=1.347 \pm$ 0.023 (stat). Multiple systematic uncertainty sources are taken into account, due to the choice of the background and signal models, to the discrepancy between data and simulation and the limited size of the simulated samples. Using the world-average values of the $\mathcal{B}\left(K^{*+} \rightarrow K_{S}^{0} \pi^{+}\right), \mathcal{B}\left(K_{S}^{0} \rightarrow \pi^{+} \pi^{-}\right)$, $\mathcal{B}\left(\bar{\Lambda} \rightarrow \bar{p} \pi^{+}\right)$branching fractions [2], the ratio $\mathcal{B}\left(B^{+} \rightarrow J / \psi \bar{\Lambda} p\right) / \mathcal{B}\left(B^{+} \rightarrow J / \psi K^{*+}\right)$ is found to be $(1.054 \pm 0.057$ (stat) \pm 0.035 (syst) $\pm 0.011(\mathcal{B})) \%$, where the third uncertainty comes from the world-average branching fractions of the decays involved. From this ratio and the world-average value of $\mathcal{B}\left(B^{+} \rightarrow J / \psi K^{*+}\right)=(1.43 \pm 0.08) \times 10^{-3}$ [2], the branching fraction $\mathcal{B}\left(B^{+} \rightarrow J / \psi \bar{\Lambda} p\right)=$ $(15.1 \pm 0.8$ (stat) \pm 0.5 (syst) $\pm 0.9(\mathcal{B})) \times 10^{-6}$ is also obtained. Furthermore the invariant mass distributions of the $J / \psi \bar{\Lambda}, J / \psi p$ and $\bar{\Lambda} p$ two-body combinations of the $B^{+} \rightarrow J / \psi \bar{\Lambda} p$ decay products have been investigated. Figure 2 shows the efficiency-corrected and sPlot backgroundsubtracted $(M(J / \psi \bar{\Lambda} p)$ being the discriminating variable) distributions of $M(J / \psi p), M(J / \psi \bar{\Lambda})$, and $M(\bar{\Lambda} p)$. None of them can be adequately described by a pure three-body non-resonant phase space decay hypothesis $\left(H_{P S}\right)$. There are, in fact, at least three known $K^{*+}$ resonances that can decay to $\bar{\Lambda} p$ and may contribute to the $M(J / \psi p)$ and $M(J / \psi \bar{\Lambda})$ distributions. To account for these possible contributions, a model independent approach developed by BaBar[11] was used. It takes into account the $K^{*+}$ introducing an angular structure into the simulated samples by applying the appropriate weights, originating from the $\cos \left(\theta_{K^{*}}\right)$ expansion in terms of Legendre polynomials:

where $\left\langle P_{j}^{N}\right\rangle=2\left\langle P_{j}^{U}\right\rangle / N_{\text {reco }}^{\text {corr }}$ are the normalised Legendre moments [8]; $N_{\text {reco }}^{\text {corr }}$ is the corrected number of reconstructed events in each $M(\bar{\Lambda} p)$ bin; $l_{\max }=8$, i.e. twice the maximum spin of the considered $K^{*+}$ resonances.

In addition the simulation is also weighted to reproduce the $M(\bar{\Lambda} p)$ spectrum observed in data, 

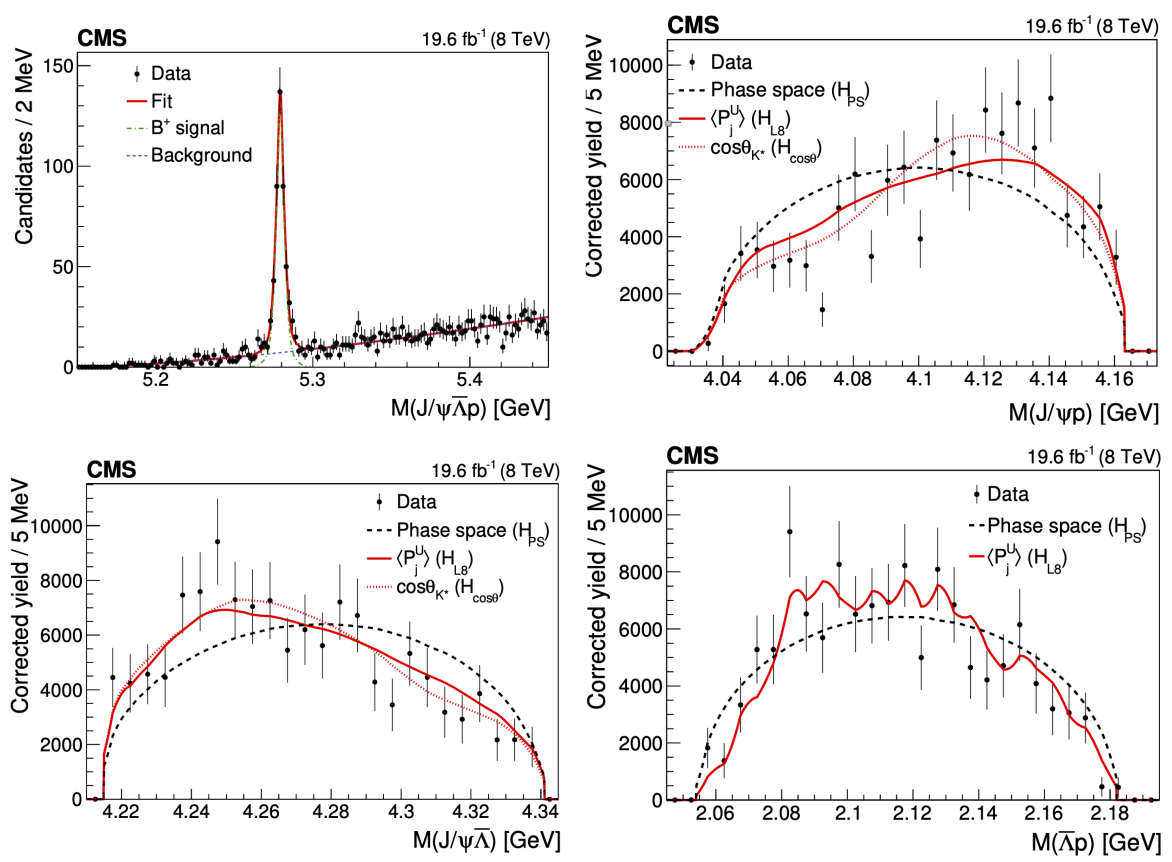

Figure 2: The invariant mass distribution of the selected $B^{+} \rightarrow J / \psi \bar{\Lambda} p$ candidates (upper left). The invariant mass distributions of the $J / \psi p$ (upper right), $J / \psi \bar{\Lambda}$ (bottom left), and $J / \psi p$ (bottom right) systems from the $B^{+} \rightarrow J / \psi p$ decay. The points show the efficiency-corrected, background-subtracted data. Superimposed curves are obtained from simulation for the different hypotheses described in the text: $H_{P S}$ (dashed lines), $H_{L 8}$ (solid curves), $H_{\cos (\theta)}$ (dotted curves).

using a linear interpolation of the data-to-simulation ratio histogram. Results of both procedures are shown in Figure 2 (solid line) and it is clear that the description of the three mass is quite improved. The compatibility of the data with both $H_{P S}$ and phase space augmented with the reweighing hypothesis $\left(H_{L 8}\right)$ is quantified using the likelihood ratio technique, using as nullhypothesis an additional hypothesis $H_{\cos (\theta)}$ that accounts for all the features in the $\cos \left(\theta_{K^{*}}\right)$ and $M(\bar{\Lambda} p)$ distributions observed in data.

Multiple systematic uncertainty contributions are taken into account, due to the choice of the background p.d.f. used in the $M(J / \psi \bar{\Lambda} p)$ fit; to the selection of the $B^{+} \rightarrow J / \psi \bar{\Lambda} p$; to the statistical fluctuations in the $2 \mathrm{D}$ efficiency calculation. The effect of the correlation between $M(\bar{\Lambda} p), \cos \left(\theta_{K^{*}}\right), M(J / \psi p)$, and $M(J / \psi \bar{\Lambda})$ is also taken into account. The significance of the incompatibility of data with the $H_{P S}$ hypothesis is then found to vary from 6.1 to $8.1,5.5$ to 7.4, and 3.4 to 4.8 standard deviations for the $J / \psi p, J / \psi \bar{\Lambda}$, and $\bar{\Lambda} p$ invariant mass distributions, respectively. The incompatibility of data with $H_{L 8}$ varies from 1.3 to 2.8 (2.7) standard deviations for the $J / \psi p(J / \psi \bar{\Lambda})$ invariant mass spectrum. This allows us to conclude that the data are consistent with the $H_{L 8}$ hypothesis.

\section{Study of excited $\Lambda_{b}^{0}$ states decaying to $\Lambda_{b}^{0} \pi^{+} \pi^{-}$}

Spectroscopy of baryons that contain a heavy-flavor quark, such as the $\Lambda_{b}^{0}$ baryon, is a very important probe to test predictions of heavy-quark effective theory [12]. In [13] the CMS Collaboration reported a study of the $\Lambda_{b}^{0} \pi^{+} \pi^{-}$invariant mass distribution in the $5900 \div 6400 \mathrm{MeV}$ range. 
The ground state baryon $\Lambda_{b}^{0}$ is reconstructed via its decays into the $J / \psi \Lambda$ and $\psi(2 S) \Lambda$ channels. The analysis uses the $p p$ collision data recorded with the CMS detector in 2016-2018 at $\sqrt{s}=13 \mathrm{TeV}$ $\left(\mathcal{L}_{\text {int }}=140 \mathrm{fb}^{-1}\right)$.

The event selection begins by building a dimuon system with two opposite sign (OS) muons with $2.90<M(\mu \mu)<3.95 \mathrm{GeV}$ and which is taken to be a $J / \psi$ candidate if $M(\mu \mu)<3.4 \mathrm{GeV}$ or a $\psi(2 S)$ candidate otherwise. Also the $\psi(2 S) \rightarrow J / \psi \pi \pi \rightarrow \mu \mu \pi \pi$ channel is considered. A $\Lambda$ candidate is formed from a displaced two-prong vertex, assuming the decay $\Lambda \rightarrow p \pi$ [9]. To form the $\Lambda_{b}^{0}$ candidates, the $J / \psi$ or $\psi(2 S)$ candidate and the $\Lambda$ candidate are fit to a common vertex, with $J / \psi$ or $\psi(2 S)$ mass constraint applied. A further selection on vertex association, displacement and pointing angle is carried on and several simulated signal samples with different masses of excited $\Lambda_{b}^{0}$ states are used in the analysis to optimise the selection criteria.The $\Lambda_{b}^{0} \pi^{+} \pi^{-}$candidates are formed by combining the selected $\Lambda_{b}^{0}$ candidates with two OS tracks originating from the primary vertex. Combinations with two prompt same-sign (SS) pions are used as a control channel. To improve the $\Lambda_{b}^{0} \pi^{+} \pi^{-}$invariant mass resolution by up to $50 \%$, all tracks forming the PV and the selected $\Lambda_{b}^{0}$ candidate, taken as a single "pseudo-track", are refit to a common vertex. The observed invariant mass distribution $m_{\Lambda_{b}^{0} \pi^{+} \pi^{-}}$of the selected signal candidates near the threshold is shown in Figure 3. The best-fit signal yields are $28.4 \pm 5.8$ and $159 \pm 14$ events, the measured masses are 5912.32 \pm 0.12 (stat) $\mathrm{MeV}$ and 5920.16 \pm 0.07 (stat) $\mathrm{MeV}$ and the statistical significance of the peaks are $5.4 \div 5.7 \sigma$ and well over $6 \sigma$, for the $\Lambda_{b}(5912)^{0}$ and $\Lambda_{b}(5920)^{0}$ states, respectively.

Higher masses in the $m_{\Lambda_{b}^{0} \pi^{+} \pi^{-}}$distribution are studied as well, as shown in Figure 3. A narrow $\Lambda_{b}^{0} \pi^{+} \pi^{-}$peak at approximately $6150 \mathrm{MeV}$ is evident, consistent with an overlap of the $\Lambda_{b}(6146)^{0}$ and $\Lambda_{b}(6152)^{0}$ signals, as well as a broad enhancement in the region below $6100 \mathrm{MeV}$. None of these features are present in the SS control region. A number of cross-checks have been performed to asses that the broad enhancement is not the result of a partially reconstructed decay, such as $\Lambda_{b}(6146)^{0}$ or $\Lambda_{b}(6152)^{0}$ decay into $\Lambda_{b}^{0} \pi^{+} \pi^{-} \pi^{0}$ (with a lost $\pi^{0}$ ); or a kinematic reflection, such as some state decaying into $\Lambda_{b}^{0} K^{ \pm} \pi^{\mp}$. In addition the $2 \mathrm{D}$ distributions of the $\Lambda_{b}^{0} \pi^{+} \pi^{-}$mass versus the $\Lambda_{b}^{0} \pi^{-}$and $\Lambda_{b}^{0} \pi^{+}$masses were inspected. If the $\Lambda_{b}^{0} \pi^{ \pm}$invariant mass ranges corresponding to the $\Sigma_{b}^{+}, \Sigma_{b}^{-}, \Sigma_{b}^{*-}$ and $\Sigma_{b}^{*-}$ baryons are vetoed, both the SS and OS mass distributions do not exhibit any broad enhancement. This suggests that the broad excess might be related to the intermediate $\Sigma_{b}^{ \pm}$ and $\Sigma_{b}^{ \pm}$baryon states, although the current size of the data set does not allow this hypothesis to be properly tested.

The fit results for the yields and masses, respectively, are $301 \pm 72$ and $6073 \pm 5 \mathrm{MeV}$ for the broad enhancement, $70 \pm 35$ and $6146.5 \pm 1.9 \mathrm{MeV}$ for the $\Lambda_{b}(6146)^{0}$, and $113 \pm 35$ and $6152.7 \pm 1.1 \mathrm{MeV}$ for the $\Lambda_{b}(6152)^{0}$. The measured natural width of the broad excess is $55 \pm 11$ (stat) MeV.

Several sources of systematic uncertainties in the measured masses are considered such as the choice of the fit model, the inclusion of the broad excess in the fit, the mass range for the fit, the peaks mass resolutions, determined from simulated samples; and the knowledge of the signal natural widths, taken from the ones measured by LHCb [14].

Using the likelihood-ratio technique the one-peak versus two-peak hypotheses for the 6150 $\mathrm{MeV}$ structure have been tested and the presence of two peaks has a statistical significance of $0.4 \sigma$. The local statistical significance of the single-peak hypothesis with respect to the background-only hypothesis is found to be between $5.4 \sigma$ and $6.5 \sigma$ (changes due to the systematic uncertainties). 

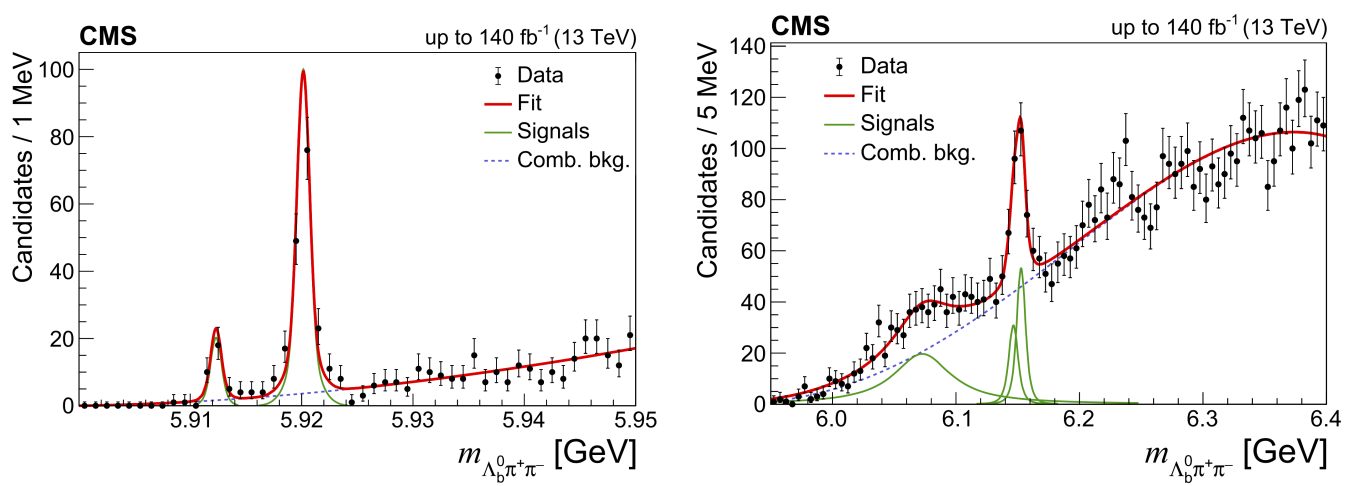

Figure 3: Invariant mass distribution of the selected $\Lambda_{b}^{0} \pi^{+} \pi$ candidates near thresh-old (left) and in the high-mass region (right). The overall fit result is shown by the thick solid line, with the thin and dashed lines representing the signal and combinatorial background components, respectively.

The broad enhancement has a local statistical significance of about $4 \sigma$. Resonances with masses between 6200 and $6400 \mathrm{MeV}$ have been also considered in the fit model and no significant excess was found. In summary the measured masses are: $M\left(\Lambda_{b}(5912)^{0}\right)=5912.32 \pm 0.12 \pm 0.01 \pm 0.17 \mathrm{MeV}$, $M\left(\Lambda_{b}(5920)^{0}\right)=5920.16 \pm 0.07 \pm 0.01 \pm 0.17 \mathrm{MeV}, M\left(\Lambda_{b}(6146)^{0}\right)=6146.5 \pm 1.9 \pm 0.8 \pm 0.2 \mathrm{MeV}$ $M\left(\Lambda_{b}(6152)^{0}\right)=6152.7 \pm 1.1 \pm 0.4 \pm 0.2 \mathrm{MeV}$, where the first uncertainty is statistical, the second is systematic and the third is the uncertainty in the world-average $\Lambda_{b}^{0}$ mass [2].

\section{References}

[1] CMS Collaboration, Measurement of the $\Upsilon(1 S)$ pair production cross section and search for resonances decaying to $\Upsilon(1 S) \mu^{+} \mu^{-}$in proton-proton collisions at $\sqrt{s}=13 \mathrm{TeV}$, Phys. Lett. $B$ 808 (2020), 135578

[2] Particle Data Group, Review of Particle Physics, Phys. Rev. D 98 (2018) no.3, 030001

[3] CMS Collaboration, Search for Higgs boson pair production in the $\gamma \gamma \mathrm{b} \overline{\mathrm{b}}$ final state in $p p$ collisions at $\sqrt{s}=13 \mathrm{TeV}$, Phys. Lett. B 788 (2019), 7-36

[4] ATLAS, CMS and LHC Higgs Combination Group, Procedure for the LHC Higgs boson search combination in summer 2011, ATL-PHYS-PUB-2011-011.

[5] G. Cowan, K. Cranmer, E. Gross and O. Vitells, Asymptotic formulae for likelihood-based tests of new physics, Eur. Phys. J. C 71 (2011), 1554

[6] T. Sjöstrand, S. Ask, J. R. Christiansen, R. Corke, N. Desai, P. Ilten, S. Mrenna, S. Prestel, C. O. Rasmussen and P. Z. Skands, Comput. Phys. Commun. 191 (2015), 159-177 doi:10.1016/j.cpc.2015.01.024 [arXiv:1410.3012 [hep-ph]].

[7] Jeffrey Davis ,Jeffrey (Heshy) Roskes,Ulascan Sarica,Markus Schulze https://spin.pha.jhu.edu

[8] A. M. Sirunyan et al. [CMS], Study of the $\mathrm{B}^{+} \rightarrow \mathrm{J} / \psi \bar{\Lambda} \mathrm{p}$ decay in proton-proton collisions at $\sqrt{s}=8 \mathrm{TeV}$, JHEP 12 (2019), 100 
[9] CMS Collaboration, CMS Tracking Performance Results from Early LHC Operation, Eur. Phys. J. C 70 (2010), 1165-1192

[10] M. Pivk and F. R. Le Diberder, SPlot: A Statistical tool to unfold data distributions, Nucl. Instrum. Meth. A 555 (2005), 356-369

[11] B. Aubert et al. [BaBar], Search for the Z(4430)- at BABAR, Phys. Rev. D 79 (2009), 112001

[12] Y. Chen, A. Fujimori, T. Müller, W. Stwalley, J. Yang (Eds.), Heavy Quark Effective Theory, Springer, Berlin, Heidelberg, 2004.

[13] CMS Collaboration, Study of excited $\Lambda_{\mathrm{b}}^{0}$ states decaying to $\Lambda_{\mathrm{b}}^{0} \pi^{+} \pi^{-}$in proton-proton collisions at $\sqrt{s}=13 \mathrm{TeV}$, Phys. Lett. B $\mathbf{8 0 3}$ (2020), 135345

[14] LHCb Collaboration, Observation of excited $\Lambda_{b}^{0}$ baryons, Phys. Rev. Lett. 109 (2012), 172003 